\title{
Frontiers in Invertebrate Physiology - grand challenge
}

\author{
Sylvia Anton ${ }^{1,2}$, Christophe Gadenne ${ }^{2}$ and Frédéric Marion-Poll ${ }^{1,3}$ \\ UMR No. 1272 INRA-UPMC, PISC, INRA Centre de Versailles, Versailles, France \\ 2 Laboratoire RCIM, UFR Sciences, Université d'Angers, Angers, France \\ ${ }^{3}$ AgroParisTech, Département Sciences de la Vie et Santé, Paris, France \\ *Correspondence: sylvia.anton@versailles.inra.fr
}

"Curse the blasted, jelly boned swines, the slimy, the belly wriggling invertebrates, the miserable sodding rotters, the flaming sods, the sniveling, dribbling, dithering palsied pulse-less lot that make up England today. They have got white of egg in their veins, and their spunk is that watery it is a marvel they can breed. They can nothing but frog-spawn - the gibberers! God, how I hate them! God curse them, funkers. God blast them, wish-wash. Exterminate them, slime. I could curse for hours and hours - God help me."

D. H. Lawrence, Letter to Edward Garnett, July 3, 1912, after the rejection of Sons and Lovers by Heinemann.

Invertebrates have long been a symbol of the evil, but the increasing knowledge accumulated by many curious scientists has changed our view profoundly. Studying invertebrate physiology is now an exciting domain, because it provides on one hand insight into general principles of animal physiology, utilizing models with different degrees of complexity and on the other hand it allows studying evolutionary adaptations to a multitude of different lifestyles. Environmental constraints and basic construction principles have lead to an amazing variation of physiological solutions to breathe, to ingest and digest food, to reproduce and to communicate, and all this on the basis of a wide range of anatomical construction principles. Therefore a comparative approach to invertebrate physiology is extremely rich and can only be encouraged. The goal of Frontiers in Invertebrate Physiology is to cover a wide range of approaches from the molecular to the cellular, organismic and even the population level. Studies on model and non-model organisms and on all aspects of physiology will be published, to provide a forum for exchange of recent advances in the field.

Invertebrate species represent $95 \%$ of all living animals. They have colonized all habitats on earth, including polar regions, deserts, and, seas. Their external skeleton (at least for some of the most prominent invertebrate groups) and their segmented central nervous system make them unique models to study developmental physiology (e.g., molting processes) and the gradual architectural evolution of their central nervous system, and the resulting neural and sensory physiology. Moreover, many invertebrate species are organized in very sophisticated societies, thus offering exciting challenges to study the physiology of intra- and interspecific interactions and their adaptation to environmental constraints (Woodard et al., 2011).

One of the major challenges for invertebrate physiology is to integrate recent advances at the molecular level into general models of physiological function. The description of complete genomes is now available for several invertebrate models such as the nematode Caenorhabditis elegans (C. elegans Sequencing Consortium, 1998), the crustacean Daphnia pulex (Colbourne et al., 2011), 12 species of Drosophila (Drosophila 12 Genomes Consortium et al., 2007), the honeybee (Weinstock et al., 2006), the malaria mosquito Anopheles gambiae (Holt et al., 2002), the pea aphid Acyrthosiphon pisum (Richards et al., 2010), and the availability of genetic tools allows now to link genes and the proteins they code for to specific physiological functions. Thus using comparative genomics, we should be able to unveil mechanisms at the cellular level, which allow invertebrates to colonize and adapt to numerous habitats and to growing anthropic pressure. We should, however, also take non-model organisms into consideration, in times where sequencing is not any more a limiting factor and where many molecular tools are adaptable to any kind of organism. The link between molecular, cellular and organismic investigation levels seems essential to provide a complete picture of physiological function today. Integrative approaches (system biology), including mathematical models will therefore play an important role.
Another issue, which has been increasing enormously during the last years, is the investigation of plasticity in the nervous control of physiological functions (Yamada et al., 2010). Adaptations to external and internal modifications in sensory and motor systems controlling different physiological functions become more and more evident. Detection of intra- and inter-specific stimuli is modified by, e.g., experience and reproductive state (Iyengar et al., 2010). However, we are far from understanding the cellular mechanisms and signaling pathways involved in these forms of physiological plasticity. It will be important in the future to investigate the role of hormones, neuromodulators and their mechanisms of action ideally in parallel in different invertebrate species both during development and in adult organisms. New biochemical techniques allow us to detect and measure minute traces of neuropeptides in single cells, whose roles are still largely unknown (Yew et al., 2009). Another important point is the rapidly developing field of research on post-transcriptional regulation mechanisms involving non-coding RNAs like microRNA (Vo et al., 2010). In addition to plasticity of the individual, different levels of control of physiological plasticity should be taken into consideration at the populational level. Here genetic variation, epigenetic phenomena, and phenotypic plasticity might play together (EspinosaSoto et al., 2011).

Politically important issues such as environmental and climatic changes (global warming) have a severe impact on the physiology of invertebrate organisms. Classically insects, but also aquatic invertebrates have been, and are still, important indicators of pollution. The typically used models, such as the honeybee, allow to study the effects of, e.g., pesticides at multiple levels, from gene expression, over ion channel function to the physiology of the nervous system, behavior, and cognition. Thus ecophysiology is a growing field within invertebrate physiology and environmental effects on, 
e.g., development, sensory and nervous systems, respiration, and feeding should be increasingly studied in the years to come.

Although the main scope of Frontiers in Invertebrate Physiology is to deal with fundamental research, contributions concerning applied aspects of invertebrate physiology are important too. Many invertebrates are of direct or indirect economic importance and therefore privileged models to study physiological mechanisms in order to improve food production (e.g., oyster, decapode crustaceans); to control animal/human and plant pathogens: (e.g., nematodes); disease vectors (e.g., bloodsucking insects); or to develop biological management methods for agricultural pests (e.g., nematodes, insects,...), to name just a few examples. Invertebrates already provide a food source for millions of human beings, but research on using more specifically insects as a source of protein is only recently starting (Verkerk et al., 2007). Studies of host-pathogen interactions, immunity and the physiology of resistance will not only be important for food production, but also for improving public health. Although invertebrate species have been used for medical purposes for more than 4,000 years and as models for research and teaching since the end of the eighteenth century, the development of invertebrate models for, e.g., neurodegenerative diseases (fruit flies, honeybees) is recent and in plain expansion (Bilen and Bonini, 2005). These recent developments will allow to reduce the use of mammals for medical research (e.g., drug development), important issue from an ethical and economic point of view.

\section{REFERENCES}

Bilen, J., and Bonini, N. M. (2005). Drosophila as a model for human neurodegenerative disease. Annu. Rev. Genet. 39, 153-171.

C. elegans Sequencing Consortium. (1998). Genome sequence of the nematode $C$-elegans: a platform for investigating biology. Science 282, 2012-2018.

Colbourne, J. K., Pfrender, M. E., Gilbert, D., Thomas, W. K., Tucker, A., Oakley, T. H., Tokishita, S., Aerts, A., Arnold, G. J., Basu, M. K., Bauer, D. J., Cáceres, C. E., Carmel, L., Casola, C., Choi, J. H., Detter, J. C., Dong, Q., Dusheyko, S., Eads, B. D., Fröhlich, T., Geiler-Samerotte, K. A., Gerlach, D., Hatcher, P., Jogdeo, S., Krijgsveld, J., Kriventseva, E. V., Kültz, D., Laforsch, C., Lindquist, E., Lopez, J., Manak, J. R., Muller, J., Pangilinan, J., Patwardhan, R. P., Pitluck, S., Pritham, E. J., Rechtsteiner, A., Rho, M., Rogozin, I. B., Sakarya, O., Salamov, A., Schaack, S., Shapiro, H., Shiga, Y., Skalitzky, C., Smith, Z., Souvorov, A., Sung,
W., Tang, Z., Tsuchiya, D., Tu, H., Vos, H., Wang, M., Wolf, Y. I., Yamagata, H., Yamada, T., Ye, Y., Shaw, J. R., Andrews, J., Crease, T. J., Tang, H., Lucas, S. M., Robertson, H. M., Bork, P., Koonin, E. V., Zdobnov, E. M., Grigoriev, I. V., Lynch, M., and Boore, J. L. (2011). The ecoresponsive genome of Daphnia pulex. Science 331, 555-561.

Drosophila 12 Genomes Consortium, Clark, A. G., Eisen, M. B., Smith, D. R., Bergman, C. M., Oliver, B., Markow, T.A., Kaufman, T. C., Kellis, M., Gelbart, W., Iyer, V. N., Pollard, D. A., Sackton, T. B., Larracuente, A. M., Singh, N. D., Abad, J. P., Abt, D. N., Adryan, B., Aguade, M., Akashi, H., Anderson, W.W., Aquadro, C. F., Ardell, D. H., Arguello, R., Artieri, C. G., Barbash, D. A., Barker, D., Barsanti, P., Batterham, P., Batzoglou, S., Begun, D., Bhutkar, A., Blanco, E., Bosak, S. A., Bradley, R. K., Brand, A. D., Brent, M. R., Brooks, A. N., Brown, R. H., Butlin, R. K., Caggese, C., Calvi, B. R., Bernardo de Carvalho, A., Caspi, A., Castrezana, S., Celniker, S. E., Chang, J. L., Chapple, C., Chatterji, S., Chinwalla, A., Civetta, A., Clifton, S. W., Comeron, J. M., Costello, J. C., Coyne, J. A., Daub, J., David, R. G., Delcher, A. L., Delehaunty, K., Do, C. B., Ebling, H., Edwards, K., Eickbush, T., Evans, J. D., Filipski, A., Findeiss, S., Freyhult, E., Fulton, L., Fulton, R., Garcia, A. C., Gardiner, A., Garfield, D. A., Garvin, B. E., Gibson, G., Gilbert, D., Gnerre, S., Godfrey, J., Good, R., Gotea, V., Gravely, B., Greenberg, A. J., Griffiths-Jones, S., Gross, S., Guigo, R., Gustafson, E. A., Haerty, W., Hahn, M. W., Halligan, D. L., Halpern, A. L., Halter, G. M., Han, M. V., Heger, A., Hillier, L., Hinrichs, A. S., Holmes, I., Hoskins, R. A., Hubisz, M. J., Hultmark, D., Huntley, M. A., Jaffe, D. B., Jagadeeshan, S., Jeck, W. R., Johnson, J., Jones, C. D., Jordan, W. C., Karpen, G. H., Kataoka, E., Keightley, P. D., Kheradpour, P., Kirkness, E. F., Koerich, L. B., Kristiansen, K., Kudrna, D., Kulathinal, R. J., Kumar, S., Kwok, R., Lander, E., Langley, C. H., Lapoint, R., Lazzaro, B. P., Lee, S. J., Levesque, L., Li, R., Lin, C. F., Lin, M. F., Lindblad-Toh, K., Llopart, A., Long, M., Low, L., Lozovsky, E., Lu, J., Luo, M., Machado, C. A., Makalowski, W., Marzo, M., Matsuda, M., Matzkin, L., McAllister, B., McBride, C. S., McKernan, B., McKernan, K., Mendez-Lago, M., Minx, P., Mollenhauer, M. U., Montooth, K., Mount, S. M., Mu, X., Myers, E., Negre, B., Newfeld, S., Nielsen, R., Noor, M. A., O’Grady, P., Pachter, L., Papaceit, M., Parisi, M. J., Parisi, M., Parts, L., Pedersen, J. S., Pesole, G., Phillippy, A. M., Ponting, C. P., Pop, M., Porcelli, D., Powell, J. R., Prohaska, S., Pruitt, K., Puig, M., Quesneville, H., Ram, K. R., Rand, D., Rasmussen, M. D., Reed, L. K., Reenan, R., Reily, A., Remington, K. A., Rieger, T. T., Ritchie, M. G., Robin, C., Rogers, Y. H., Rohde, C., Rozas, J., Rubenfield, M. J., Ruiz, A., Russo, S., Salzberg, S. L., Sanchez-Gracia, A., Saranga, D. J., Sato, H., Schaeffer, S. W., Schatz, M. C., Schlenke, T., Schwartz, R., Segarra, C., Singh, R. S., Sirot, L., Sirota, M., Sisneros, N. B., Smith, C. D., Smith, T. F., Spieth, J., Stage, D. E., Stark, A., Stephan, W., Strausberg, R. L., Strempel, S., Sturgill, D., Sutton, G., Sutton, G. G., Tao, W., Teichmann, S., Tobari, Y.N., Tomimura, Y., Tsolas, J. M., Valente, V. L., Venter, E., Venter, J. C., Vicario, S., Vieira, F. G., Vilella, A. J., Villasante, A., Walenz, B., Wang, J., Wasserman, M., Watts, T., Wilson, D., Wilson, R. K., Wing, R. A., Wolfner, M. F., Wong, A., Wong, G. K., Wu, C. I., Wu, G., Yamamoto, D., Yang, H. P., Yang, S. P., Yorke, J. A., Yoshida, K., Zdobnov, E., Zhang, P., Zhang, Y., Zimin, A. V., Baldwin, J., Abdouelleil, A., Abdulkadir, J., Abebe, A., Abera,
B., Abreu, J., Acer, S. C., Aftuck, L., Alexander, A., An, P., Anderson, E., Anderson, S., Arachi, H., Azer, M., Bachantsang, P., Barry, A., Bayul, T., Berlin, A., Bessette, D., Bloom, T., Blye, J., Boguslavskiy, L., Bonnet, C., Boukhgalter, B., Bourzgui, I., Brown, A., Cahill, P., Channer, S., Cheshatsang, Y., Chuda, L., Citroen, M., Collymore, A., Cooke, P., Costello, M., D’Aco, K., Daza, R., De Haan, G., DeGray, S., DeMaso, C., Dhargay, N., Dooley, K., Dooley, E., Doricent, M., Dorje, P., Dorjee, K., Dupes, A., Elong, R., Falk, J., Farina, A., Faro, S., Ferguson, D., Fisher, S., Foley, C. D., Franke, A., Friedrich, D., Gadbois, L., Gearin, G., Gearin, C. R., Giannoukos, G., Goode, T., Graham, J., Grandbois, E., Grewal, S., Gyaltsen, K., Hafez, N., Hagos, B., Hall, J., Henson, C., Hollinger, A., Honan, T., Huard, M. D., Hughes, L., Hurhula, B., Husby, M. E., Kamat, A., Kanga, B., Kashin, S., Khazanovich, D., Kisner, P., Lance, K., Lara, M., Lee, W., Lennon, N., Letendre, F., LeVine, R., Lipovsky, A., Liu, X., Liu, J., Liu, S., Lokyitsang, T., Lokyitsang, Y., Lubonja, R., Lui, A., MacDonald, P., Magnisalis, V., Maru, K., Matthews, C., McCusker, W., McDonough, S., Mehta, T., Meldrim, J., Meneus, L., Mihai, O., Mihalev, A., Mihova, T., Mittelman, R., Mlenga, V., Montmayeur, A., Mulrain, L., Navidi, A., Naylor, J., Negash, T., Nguyen, T., Nguyen, N., Nicol, R., Norbu, C., Norbu, N., Novod, N., O’Neill, B., Osman, S., Markiewicz, E., Oyono, O. L., Patti, C., Phunkhang, P., Pierre, F., Priest, M., Raghuraman, S., Rege, F., Reyes, R., Rise, C., Rogov, P., Ross, K., Ryan, E., Settipalli, S., Shea, T., Sherpa, N., Shi, L., Shih, D., Sparrow, T., Spaulding, J., Stalker, J., Stange-Thomann, N., Stavropoulos, S., Stone, C., Strader, C., Tesfaye, S., Thomson, T., Thoulutsang, Y., Thoulutsang, D., Topham, K., Topping, I., Tsamla, T., Vassiliev, H., Vo, A., Wangchuk, T., Wangdi, T., Weiand, M., Wilkinson, J., Wilson, A., Yadav, S., Young, G., Yu, Q., Zembek, L., Zhong, D., Zimmer, A., Zwirko, Z., Jaffe, D. B., Alvarez, P., Brockman, W., Butler, J., Chin, C., Gnerre, S., Grabherr, M., Kleber, M., Mauceli, E., and MacCallum, I. (2007). Evolution of genes and genomes on the Drosophila phylogeny. Nature 450, 203-218.

Espinosa-Soto, C., Martin, O. C., and Wagner, A. (2011). Phenotypic plasticity can facilitate adaptive evolution in gene regulatory circuits. BMC Evol. Biol. 11, 5. doi: 10.1186/1471-2148-11-5

Holt, R., Subramanian, G. M., Halpern, A., Sutton, G. G., Charlab, R., Nusskern, D. R., Wincker, P., Clark, A. G., Ribeiro, J. M., Wides, R., Salzberg, S. L., Loftus, B., Yandell, M., Majoros, W. H., Rusch, D. B., Lai, Z., Kraft, C. L., Abril, J. F., Anthouard, V., Arensburger, P. Atkinson, P.W., Baden, H., de Berardinis, V., Baldwin, D., Benes, V., Biedler, J., Blass, C., Bolanos, R., Boscus, D., Barnstead, M., Cai, S., Center, A., Chaturverdi, K., Christophides, G. K., Chrystal, M. A., Clamp, M., Cravchik, A., Curwen, V., Dana, A., Delcher, A., Dew, I., Evans, C. A., Flanigan, M., Grundschober-Freimoser, A., Friedli, L., Gu, Z., Guan, P., Guigo, R., Hillenmeyer, M. E., Hladun, S. L., Hogan, J. R., Hong, Y. S., Hoover, J., Jaillon, O., Ke, Z., Kodira, C., Kokoza, E., Koutsos, A., Letunic, I., Levitsky, A., Liang, Y., Lin, J. J., Lobo, N. F., Lopez, J. R., Malek, J. A., McIntosh, T. C., Meister, S., Miller, J., Mobarry, C., Mongin, E., Murphy, S. D., O’Brochta, D. A., Pfannkoch, C., Qi, R., Regier, M. A., Remington, K., Shao, H., Sharakhova, M. V., Sitter, C. D., Shetty, J., Smith, T. J., Strong, R., Sun, J., Thomasova, D., Ton, L. Q., Topalis, P., Tu, Z., Unger, M. F., Walenz, B., Wang, A., Wang, J., Wang, M., Wang, 
X., Woodford, K. J., Wortman, J. R., Wu, M., Yao, A., Zdobnov, E. M., Zhang, H., Zhao, Q., Zhao, S., Zhu, S. C., Zhimulev, I., Coluzzi, M., della Torre, A., Roth, C. W., Louis, C., Kalush, F., Mural, R. J., Myers, E. W., Adams, M. D., Smith, H. O., Broder, S., Gardner, M. J., Fraser, C. M., Birney, E., Bork, P., Brey, P. T., Venter, J. C., Weissenbach, J., Kafatos, F. C., Collins, F. H., and Hoffman, S. L. (2002). The genome sequence of the malaria mosquito Anopheles gambiae. Science 298, 129-149.

Iyengar, A., Chakraborty, T. S., Goswami, S. P., Wu, C.-F., and Siddiqi, O. (2010). Post-eclosion odor experience modifies olfactory receptor neuron coding in Drosophila. Proc. Natl. Acad. Sci. U.S.A. 107, 9855-9860.

Richards, S., Gibbs, R. A., Gerardo, N. M., Moran, N., Nakabachi, A., Stern, D., Tagu, D., Wilson, A. C. C., Muzny, D., Kovar, C., Cree, A., Chacko, J., Chandrabose, M. N., Dinh, H. H., Gabisi, R. A., Hines, S., Hume, J., Jhangian, S. N., Joshi, V., Lewis, L. R., Liu, Y. S., Lopez, J., Morgan, M. B., Nguyen, N. B., Okwuonu, G. O., Ruiz, S. J., Santibanez, J., Wright, R. A., Fowler, G. R., Hitchens, M. E., Lozado, R. J., Moen, C., Steffen, D., Warren, J. T., Zhang, J., Nazareth, L. V., Chavez, D., Davis, C., Lee, S. L., Patel, B. M., Pu, L. L., Bell, S. N., Johnson, A. J., Vattathil, S., Williams, R. L. Jr., Shigenobu, S., Stern, D., Richards, S., Dang, P. M., Morioka, M., Fukatsu, T., Kudo, T., Miyagishima, S. Y., Nakabachi, A., Jiang, H., Richards, S., Worley, K. C., Legeai, F., Gauthier, J. P., Collin, O., Shigenobu, S., Tagu, D., Legeai, F., Zhang, L., Gauthier, J. P., Shigenobu, S., Tagu, D., Richards, S., Chen, H. C., Ermolaeva, O., Hlavina, W., Kapustin, Y., Kiryutin, B., Kitts, P., Maglott, D., Murphy, T., Pruitt, K., Sapojnikov, V., Souvorov, A., Thibaud-Nissen, F. F., Câmara, F., Guigó, R., Stanke, M., Solovyev, V., Kosarev, P., Gilbert, D., Gabaldón, T., Huerta-Cepas, J., Marcet-Houben, M., Pignatelli, M., Gilbert, D., Moya, A., Rispe, C., Ollivier, M., Legeai, F., Tagu, D., Quesneville, H., Permal, E., Moya, A., Llorens, C., Futami, R., Wilson, A. C., Hedges, D., Robertson, H. M., Alioto, T., Mariotti, M., Guigó, R., Nikoh, N., McCutcheon, J. P., Pignatelli, M., Burke, G., Gerardo, N. M., Kamins, A., Latorre, A., Moya, A., Kudo, T., Miyagishima, S. Y., Moran, N. A., Nakabachi, A., Ashton, P., Calevro, F., Charles, H., Colella, S., Douglas, A., Jander, G., Jones, D. H., Febvay, G., Kamphuis, L. G., Kushlan, P. F., Macdonald, S., Ramsey, J., Schwartz, J., Seah, S., Thomas, G., Vellozo, A., Wilson, A. C., Shigenobu, S., Richards, S., Moran, N., Miyagishima, S. Y., Nakabachi, A., Cass, B., Degnan, P., Hurwitz, B., Leonardo, T., Koga, R., Moran, N., Richards, S., Stern, D., Altincicek, B., Anselme, C., Atamian, H., Barribeau, S. M., de Vos, M., Duncan, E. J., Evans, J., Gabaldon, T., Gerardo, N. M., Ghanim, M., Heddi, A., Kaloshian, I., Latorre, A., Vincent-Monegat, C., Moya, A., Nakabachi, A., Parker, B. J., Pérez-Brocal, V., Pignatelli, M., Rahbé, Y., Ramsey, J., Spragg, C. J., Tamames, J., Tamarit, D., Tamborindeguy, C., Vilcinskas, A., Shigenobu, S., Bickel, R. D., Brisson, J. A., Butts, T., Chang, C. C., Christiaens, O., Davis, G. K., Duncan, E., Ferrier, D., Iga, M., Janssen, R., Lu, H. L., McGregor, A., Miura, T., Smagghe, G., Smith, J., van der Zee, M., Velarde, R., Wilson, M., Dearden, P., Stern, D., Chang, C. C., Lu, H. L., Bickel, R. D., Shigenobu, S., Davis, G. K., Brisson, J. A., Edwards, O. R., Gordon, K., Hilgarth, R. S., Rider, S. D. Jr., Robertson, H.M., Srinivasan, D., Walsh, T. K., Brisson, J. A., Ishikawa, A., Miura, T., Miura, T., Brisson, J. A.,
Ishikawa, A., Jaubert-Possamai, S., Tagu, D., Walsh, T. K., Srinivasan, D., Fenton, B., Jaubert-Possamai, S., Huang, W., Jones, D. H., Wilson, A. C., Legeai, F., Walsh, T. K., Rizk, G., Edwards, O. R., Gordon, K., Lavenier, D., Nicolas, J., Tagu, D., Jaubert-Possamai, S., Rispe, C., Smadja, C., Robertson, H. M., Zhou, J. J., Vieira, F. G., Smadja, C., He, X. L., Liu, R., Rozas, J., Field, L. M., Rider, S. D. Jr., Ramsey, J., Gordon, K., Walsh, T. K., de Vos, M., Jander, G., Ashton, P. D., Campbell, P., Carolan, J. C., Douglas, A. E., Edwards, O. R., Fitzroy, C. I., Kamphuis, L. G., Reardon, K. T., Reeck, G., Singh, K., Wilkinson, T. L., Huybrechts, J., Abdel-latief, M., Robichon, A., Veenstra, J. A., Hauser, F., Cazzamali, G., Schneider, M., Williamson, M., Stafflinger, E., Hansen, K. K., Grimmelikhuijzen, C. J., Tagu, D., Price, D. R., Caillaud, M., van Fleet, E., Ren, Q., Rahbé, Y., Douglas, A. E., Gatehouse, J. A., Brault, V., Monsion, B., Caillaud, M., Van Fleet, E., Diaz, J., Hunnicutt, L., Nakabachi, A., Ju, H. J., Tamborindeguy, C., Pechuan, X., Aguilar, J., Tamarit, D., Llorens, C., Moya, A., Nakabachi, A., Miyagishima, S. Y., Cortés, T., Ortiz-Rivas, B., Martínez-Torres, D., Rispe, C., Dombrovsky, A., Jaubert-Possamai, S., Tagu, D., Nakabachi, A., Shigenobu, S., Miyagishima, S. Y., Channels, I., Dale, R. P., Walsh, T. K., Tamborindeguy, C., Davies, T. G., Field, L. M., Williamson, M. S., Jones, A., Sattelle, D., Williamson, S., Wolstenholme, A., Campbell, P., Carolan, J. C., Edwards, O. R., Gordon, K., Llorens, C., Moya, A., Pignatelli, M., Rahbé, Y., Rispe, C., Reeck, G. R., Vellozo, A., Colella, S., Cottret, L., Febvay, G., Calevro, F., Rahbé, Y., Douglas, A., Sagot, M. F., Charles, H., Rispe, C., Heckel, D. G., and Hunter, W. (2010). Genome sequence of the pea aphid Acyrthosiphon pisum. PLoS Biol. 8, e1000313. doi: 10.1371/journal.pbio.1000313

Verkerk, M. C., Tramper, J., van Trijp, J. C. M., and Martens, D. E. (2007). Insect cells for human food. Biotechnol. Adv. 25, 198-202.

Vo, N. K., Cambronne, X. A., and Goodman, R. H. (2010). MicroRNA pathways in neural development and plasticity. Curr. Opin. Neurobiol. 20, 457-465.

Weinstock, G. M., Robinson, G. E., Gibbs, R. A., Worley, K. C., Evans, J. D., Maleszka, R., Robertson, H. M., Weaver, D. B., Beye, M., Bork, P., Elsik, C. G., Evans, J. D., Hartfelder, K., Hunt, G. J., Robertson, H. M., Robinson, G. E., Maleszka, R., Weinstock, G. M., Worley, K. C., Zdobnov, E. M., Hartfelder, K., Amdam, G. V., Bitondi, M. M., Collins, A. M., Cristino, A. S., Evans, J.D., Lattorff, M. G., Lobo, C.H., Moritz, R. F., Nunes, F. M., Page, R. E. Jr., Simões, Z. L., Wheeler, D., Carninci, P., Fukuda, S., Hayashizaki, Y., Kai, C., Kawai, J., Sakazume, N., Sasaki, D., Tagami, M., Maleszka, R., Amdam, G. V., Albert, S., Baggerman, G., Beggs, K. T., Bloch, G., Cazzamali, G., Cohen, M., Drapeau, M. D., Eisenhardt, D., Emore, C., Ewing, M. A., Fahrbach, S. E., Forêt, S., Grimmelikhuijzen, C. J., Hauser, F., Hummon, A. B., Hunt, G. J., Huybrechts, J., Jones, A. K., Kadowaki, T., Kaplan, N., Kucharski, R., Leboulle, G., Linial, M., Littleton, J. T., Mercer, A. R., Page, R. E. Jr., Robertson, H. M., Robinson, G. E., Richmond, T. A., RodriguezZas, S. L., Rubin, E. B., Sattelle, D. B., Schlipalius, D., Schoofs, L., Shemesh, Y., Sweedler, J. V., Velarde, R., Verleyen, P., Vierstraete, E., Williamson, M. R., Beye, M., Ament, S. A., Brown, S. J., Corona, M., Dearden, P. K., Dunn, W. A., Elekonich, M. M., Elsik, C. G., Forêt, S., Fujiyuki, T., Gattermeier, E., Gempe, T., Hasselmann, M., Kadowaki, T., Kage, E., Kamikouchi, A., Kubo, T., Kucharski, R., Kunieda, T., Lorenzen, M.,
Maleszka, R., Milshina, N. V., Morioka, M., Ohashi, K., Overbeek, R., Page, R. E. Jr., Robertson, H. M., Robinson, G. E., Ross, C. A., Schioett, M., Shippy, T., Takeuchi, H., Toth, A. L., Willis, J. H., Wilson, M. J., Robertson, H. M., Zdobnov, E. M., Bork, P., Elsik, C. G., Gordon, K. H., Letunic, I., Hackett, K., Peterson, J., Felsenfeld, A., Guyer, M., Solignac, M., Agarwala, R., Cornuet, J. M., Elsik, C. G., Emore, C., Hunt, G. J., Monnerot, M., Mougel, F., Reese, J. T., Schlipalius, D., Vautrin, D., Weaver, D. B., Gillespie, J. J., Cannone, J. J., Gutell, R. R., Johnston, J.S., Elsik, C. G., Cazzamali, G., Eisen, M. B., Grimmelikhuijzen, C. J., Hauser, F., Hummon, A. B., Iyer, V. N., Iyer, V., Kosarev, P., Mackey, A. J., Maleszka, R., Reese, J. T., Richmond, T. A., Robertson, H. M., Solovyev, V., Souvorov, A., Sweedler, J. V., Weinstock, G. M., Willliamson, M. R., Zdobnov, E. M., Evans, J. D., Aronstein, K. A., Bilikova, K., Chen, Y. P., Clark, A. G., Decanini, L. I., Gelbart, W. M., Hetru, C., Hultmark, D., Imler, J. L., Jiang, H., Kanost, M., Kimura, K., Lazzaro, B. P., Lopez, D. L., Simuth, J., Thompson, G. J., Zou, Z., De Jong, P., Sodergren, E., Csûrös, M., Milosavljevic, A., Johnston, J. S., Osoegawa, K., Richards, S., Shu, C. L., Weinstock, G. M., Elsik, C. G., Duret, L., Elhaik, E., Graur, D., Reese, J. T., Robertson, H. M., Robertson, H. M., Elsik, C. G., Maleszka, R., Weaver, D. B., Amdam, G. V., Anzola, J. M., Campbell, K. S., Childs, K. L., Collinge, D., Crosby, M. A., Dickens, C. M., Elsik, C. G., Gordon, K. H., Grametes, L. S., Grozinger, C. M., Jones, P. L., Jorda, M., Ling, X., Matthews, B. B., Miller, J., Milshina, N. V., Mizzen, C., Peinado, M. A., Reese, J. T., Reid, J. G., Robertson, H. M., Robinson, G. E., Russo, S. M., Schroeder, A. J., St, P.ierre, S. E., Wang, Y., Zhou, P., Robertson, H. M., Agarwala, R., Elsik, C. G., Milshina, N. V., Reese, J. T., Weaver, D. B., Worley, K. C., Childs, K. L., Dickens, C. M., Elsik, C. G., Gelbart, W. M., Jiang, H., Kitts, P., Milshina, N. V., Reese, J. T., Ruef, B., Russo, S. M., Venkatraman, A., Weinstock, G. M., Zhang, L., Zhou, P., Johnston, J. S., Aquino-Perez, G., Cornuet, J. M., Monnerot, M., Solignac, M., Vautrin, D., Whitfield, C. W., Behura, S., Berlocher, S. H., Clark, A. G., Gibbs, R. A., Johnston, J. S., Sheppard, W. S., Smith, D. R., Suarez, A. V., Tsutsui, N. D., Weaver, D. B., Wei, X., Wheeler, D., Weinstock, G. M., Worley, K. C., Havlak, P., Li, B., Liu, Y., Sodergren, E., Zhang, L., Beye, M., Hasselmann, M., Jolivet, A., Lee, S., Nazareth, L. V., Pu, L. L., Thorn, R., Weinstock, G. M., Stolc, V., Robinson, G. E., Maleszka, R., Newman, T., Samanta, M., Tongprasit, W. A., Aronstein, K. A., Claudianos, C., Berenbaum, M. R., Biswas, S., de Graaf, D. C., Feyereisen, R., Johnson, R. M., Oakeshott, J. G., Ranson, H., Schuler, M. A., Muzny, D., Gibbs, R. A., Weinstock, G. M., Chacko, J., Davis, C., Dinh, H., Gill, R., Hernandez, J., Hines, S., Hume, J., Jackson, L., Kovar, C., Lewis, L., Miner, G., Morgan, M., Nazareth, L. V., Nguyen, N., Okwuonu, G., Paul, H., Richards, S., Santibanez, J., Savery, G., Sodergren, E., Svatek, A., Villasana, D., and Wright, R. (2006). Insights into social insects from the genome of the honeybee Apis mellifera. Nature 443, 931-949.

Woodard, S. H., Fischman, B. J., Venkat, A., Hudson, M.E., Varala, K., Cameron, S.A., Clark, A. G., and Robinson, G. E. (2011). Genes involved in convergent evolution of eusociality in bees. Proc. Natl. Acad. Sci. U.S.A. 108, 7472-7477.

Yamada, K., Hirotsu, T., Matsuki, M., Butcher, R. A., Tomioka, M., Ishihara, T., Clardy, J., Kunitomo, H., and Iino, Y. (2010). Olfactory plasticity is regulated 
by pheromonal signaling in Caenorhabditis elegans. Science 329, 1647-1650.

Yew, J. Y., Wang, Y., Barteneva, N., Dikler, S., Kutz-Naber, K. K., Li, L., and Kravitz, E.A. (2009). Analysis of neuropeptide expression and localization in adult Drosophila melanogaster central nervous system by affinity cell-capture mass spectrometry. J. Proteome Res. 8, 1271-1284.
Received: 01 July 2011; accepted: 01 July 2011; published online: 18 July 2011.

Citation: Anton S, Gadenne C and Marion-Poll F (2011) Frontiers in Invertebrate Physiology - grand challenge. Front. Physio. 2:38. doi: 10.3389/fphys.2011.00038

This article was submitted to Frontiers in Invertebrate Physiology, a specialty of Frontiers in Physiology.
Copyright () 2011 Anton, Gadenne and Marion-Poll. This is an open-access article subject to a non-exclusive license between the authors and Frontiers Media SA, which permits use, distribution and reproduction in other forums, provided the original authors and source are credited and other Frontiers conditions are complied with. 